\title{
The $\tau$-fixed point property for left reversible semigroups
}

\author{
Francisco E Castillo-Santos ${ }^{1}$ and Maria A Japón²*
}

\author{
*Correspondence: japon@us.es \\ ${ }^{2}$ Departamento de Análisis \\ Matemático, Facultad de \\ Matemáticas, Universidad de Sevilla, \\ Tarfia s/n, Sevilla, 41012, Spain \\ Full list of author information is \\ available at the end of the article
}

\begin{abstract}
In this article we use the generalized Gossez-Lami Dozo property and the Opial condition to study the fixed point property for left reversible semigroups in separable Banach spaces. As a consequence, some previous results will be deduced and new examples of Banach spaces satisfying the fixed point property for left reversible semigroups are shown. We will also extend some previous theorems when we consider the semigroup formed by a unique nonexpansive mapping and its iterates.
\end{abstract}

MSC: 46B03; 47H09; 47H10

Keywords: Schauder basis; sequentially separating norms; fixed point property; nonexpansive mappings; renorming theory; Schur property

\section{Introduction}

A semigroup $S$ is said to be a semitopological semigroup if $S$ is equipped with a Hausdorff topology such that for each $a \in S$, the two mappings from $S$ into $S$ defined by $s \rightarrow$ as and $s \rightarrow s a$ are continuous. A semitopological semigroup $S$ is said to be left reversible if any two nonempty closed right ideals of $S$ have nonempty intersection. Clearly every Abelian semitopological semigroup and every semitopological group are left reversible. Also left amenable and in particular amenable semitopological semigroups are left reversible [1].

Let $C$ be a subset of a Banach space $X$ and let $S$ be a semitopological semigroup. A nonexpansive action of $S$ on the set $C$ is a map $\phi: S \times C \rightarrow C$, denoted by $\phi(s, u)=s(u)$ (or $s u$ ), which satisfies:

(i) $t s(u)=t(s u)$ for all $t, s \in S$ and $u \in C$.

(ii) For all $u_{0} \in C$, the function $s \in S \rightarrow s\left(u_{0}\right) \in C$ is continuous.

(iii) For every $s \in S$, the mapping $u \in C \rightarrow s(u) \in C$ is nonexpansive.

A subset $C$ is said to verify the fixed point property for left reversible semigroups if for every left reversible semitopological semigroup $S$ and for every nonexpansive action $\phi: S \times C \rightarrow C$, the set $\operatorname{Fix}(S):=\{u \in C: t(u)=u, \forall t \in S\}$ is nonempty.

Definition 1.1 Let $X$ be a Banach space and $\tau$ be a topology on $X$. It is said that $X$ has the $\tau$ fixed point property ( $\tau$-FPP) for left reversible semigroups if every closed, convex, bounded subset $C$ which is $\tau$-compact has the fixed point property for left reversible semigroups.

Given a nonexpansive mapping $T$, if we replace the left reversible semigroup by the discrete and Abelian semigroup $\left\{T, T^{2}, T^{3}, \ldots\right\}$ acting from $C$ to $C$, Definition 1.1 becomes the

(c) 2015 Castillo-Santos and Japón. This article is distributed under the terms of the Creative Commons Attribution 4.0 International License (http://creativecommons.org/licenses/by/4.0/), which permits unrestricted use, distribution, and reproduction in any medium, provided you give appropriate credit to the original author(s) and the source, provide a link to the Creative Commons license, and indicate if changes were made. 
usual definition of the $\tau$-FPP for nonexpansive mappings. There exist some Banach spaces failing the $w$-FPP [2] and therefore they fail the $w$-FPP for left reversible semigroups (we can consider the semigroup $S=\left\{T, T^{2}, T^{3}, \ldots\right\}$ where $T$ is the fixed point free nonexpansive mapping in the well-known Alspach example [2]).

In 1965 Kirk proved that every Banach space with weak normal structure satisfies the $w$-FPP for nonexpansive mappings. In a similar way it can be proved that weak* normal structure implies the weak*-FPP in dual Banach spaces.

In the seventies Kirk's result was generalized by Lim [3], Holmes and Lau [4] in the setting of nonexpansive actions of left reversible semigroups, that is, weak normal structure implies the $w$-FPP for left reversible semigroups. In the case of dual Banach spaces, such a general statement is still unknown for the weak* normal structure and the weak*-fixed point property for left reversible semigroups (see Open Problem 6.3 in [5]).

Particular examples of dual Banach spaces are known to satisfy the weak*-FPP for left reversible semigroups. In $1980 \mathrm{Lim}$ [6] proved that the sequence space $\ell_{1}$ satisfies the weak*-FPP for left reversible semigroups. In 2010, Lau and Mah in [5] generalized Lim's result by proving that the Fourier-Stieltjes algebra $B(G)$ of a separable compact group verifies the weak*-FPP for left reversible semigroups. Notice that if $G$ is the torus group, then $B(G)$ is isometric to $\ell_{1}(\mathbb{Z})$. In 2010, Randrianantoanina [7] proved that the space $\mathcal{T}(H)$ of trace class operators on a Hilbert space also satisfies the weak*-FPP for left reversible semigroups. He also proved the same property for the Hardy Banach space [8].

However, the techniques used in the previous articles cannot be extended to more general dual Banach spaces since they are mainly based on the following fact: in the abovementioned Banach spaces, the asymptotic center of a weak* compact set with respect to a decreasing net of bounded subsets is proved to be either norm compact or weakly compact. This is not true for every weak* compact set in a dual Banach space, as we will later check in Example 2.1.

In 2010, Randrianantoanina [7] proved that the Banach space $L_{1}[0,1]$ or, more generally, every noncommutative $L_{1}$-space associated to a finite von Neumann algebra satisfies the fixed point property for left reversible semigroups with respect to the abstract measure topology $\tau$ (the convergence in measure topology in case of $L_{1}[0,1]$ ). Here the asymptotic centers of $\tau$-compact sets are norm compact.

In this paper we develop new arguments to deduce whether a dual Banach space satisfies the weak*-FPP for left reversible semigroups. More generally, we will consider $\tau$ as any translation invariant topology on a separable Banach space $X$ and we give sufficient conditions to assure the $\tau$-FPP for left reversible semigroups. The strict Opial condition and the generalized Gossez-Lami Dozo property will be our main tools. Most of the previous known results will be deduced from ours, but we will also achieve new examples of Banach spaces which satisfy the $\tau$-FPP for left reversible semigroups. Here we will consider different types of topologies. Firstly we will regard the weak* topology in Musielak-Orlicz sequence spaces, in some renormings of $\ell_{1}$ and in some other dual Banach spaces nonisomorphic to $\ell_{1}$. We will also consider the topology of the convergence locally in measure in some function spaces, the abstract measure topology in $L$-embedded Banach spaces and the topology of $\rho$-almost everywhere convergence in modular function spaces.

Moreover, we will extend some known results for nonexpansive mappings to the setting of the fixed point property for left reversible semigroups. 


\section{Preliminaries}

We introduce some definitions and concepts.

Let $X$ be a Banach space and let $\left\{B_{s}\right\}_{s \in A}$ be a decreasing net of bounded subsets of $X$. For $x \in X$ and $s \in A$, we consider

$$
\begin{aligned}
& r_{s}(x):=\sup \left\{\|x-y\|: y \in B_{s}\right\}, \\
& r(x):=\inf \left\{r_{s}(x): s \in A\right\}=\lim _{s} r_{s}(x) .
\end{aligned}
$$

Notice that $r(\cdot)$ is a continuous function for the norm topology.

We defined the asymptotic radius and the asymptotic center of a set $C$ with respect to the family $\left\{B_{s}\right\}_{s \in A}$ as

$$
\begin{aligned}
& r_{0}:=\inf \{r(x): x \in C\}, \\
& \mathcal{A C}\left(\left\{B_{s}\right\}_{s \in A}, C\right):=\left\{x \in C: r(x)=r_{0}\right\} .
\end{aligned}
$$

In the following example we check that asymptotic centers of weak*-compact sets are not weakly compact in general.

Example 2.1 Let $X$ be the space $\ell_{1}$ renormed as follows:

$$
\|x\|=\max _{n \in \mathbb{N}}\left(|x(n)|+\frac{1}{2} \sum_{i=n+1}^{\infty}|x(i)|\right) .
$$

Since $\left\{e_{n}\right\}$ is a monotonous boundedly complete Schauder basis for $X=\left(\ell_{1},\|\cdot\|\right)$, this space is isometric to the dual of the closed subspace spanned by the orthogonal functions $\left\{e_{n}^{*}\right\}$. Thus $X$ is a dual space and in fact its weak* topology coincides with the $\sigma\left(\ell_{1}, c_{0}\right)$ topology.

Consider the set

$$
C:=\left\{\sum_{n=1}^{\infty} t_{n} e_{n}: t_{n} \geq 0, \sum_{n=1}^{\infty} t_{n} \leq \frac{1}{2}\right\},
$$

which is a closed convex bounded $w^{*}$-compact set.

Define the bounded subsets $B_{s}=\left\{e_{k}: k \geq s\right\}$, where the $\left\{e_{n}\right\}$ are the unit basic vectors. In this case $r(x)=\lim \sup _{s}\left\|x-e_{s}\right\|$, and it is easy to check that $r(x) \geq 1$ for all $x \in \ell_{1}$. However, for all $k, n$ with $k<n$,

$$
\left\|\frac{1}{2} e_{k}-e_{n}\right\|=1
$$

which implies that $\frac{1}{2} e_{n}$ belongs to the asymptotic center of $C$ with respect to the sequence $\left\{B_{s}\right\}_{s}$, and this center is not weakly compact. Therefore, the arguments used in [6-8] or [5] to prove the existence of a common fixed point for left reversible semigroups of nonexpansive mappings are not useful in this example. We will later deduce that $\left(\ell_{1},\|\cdot\|\right)$ does satisfy the $w^{*}$-FPP for left reversible semigroups.

Let $(T, \tau)$ be a topological space. Recall that a function $f: T \rightarrow \mathbb{R}$ is said to be $\tau$-sequentially lower semicontinuous $\left(\tau\right.$-slsc) if $f(t) \leq \liminf _{n} f\left(t_{n}\right)$ for every sequence $\left(t_{n}\right)_{n} \subset T$ 
with $\tau-\lim _{n} t_{n}=t_{0}$. From now on, let $X$ be a Banach space and let $\tau$ be a translation invariant topology on $X$, that is, $\tau-\lim _{n} x_{n}=x$ if and only if $\tau-\lim _{n}\left(x_{n}-x\right)=0$.

We introduce the following definitions which generalize two geometric properties which are well known in case of the weak topology. These properties were attempts to get some information about the behavior of the norm on the weakly convergent sequences. The Opial condition was introduced by Opial (1967) [9] and the generalized Gossez-Lami Dozo property was introduced by Jiménez-Melado (1992) [10] when $\tau$ is the weak topology.

Definition 2.2 Let $(X,\|\cdot\|)$ be a Banach space and $\tau$ be a topology on $X$. We will say that $X$ has the generalized Gossez-Lami Dozo property for the topology $\tau$ ( $\tau$-GGLD) if for every (norm) bounded and $\tau$-null sequence $\left\{x_{n}\right\}$ such that $\lim \left\|x_{n}\right\| \neq 0$ and $\lim _{n, m, n \neq m}\left\|x_{n}-x_{m}\right\|$ exists, it is the case that

$$
\lim \left\|x_{n}\right\|<\lim _{n, m, n \neq m}\left\|x_{n}-x_{m}\right\| .
$$

Definition 2.3 It is said that a Banach space $(X,\|\cdot\|)$ satisfies the Opial condition with respect to a topology $\tau$ if

$$
\liminf _{n}\left\|x_{n}-x_{0}\right\|<\liminf _{n}\left\|x_{n}-x\right\|
$$

for all $x \in X$ with $x \neq x_{0}$, whenever $\left(x_{n}\right)$ is a sequence in $X$ with $\tau-\lim _{n} x_{n}=x_{0}$.

The $\tau$-GGLD property and the Opial condition will be the key to our main results. It is well known that these properties are not related. We will also illustrate this assertion with some examples.

Definition 2.4 Let $(X,\|\cdot\|)$ be a Banach space and $\tau$ be a topology on $X$. It is said that $X$ is uniformly Kadec-Klee with respect to $\tau, \operatorname{UKK}(\tau)$, if for every $\epsilon>0$ there exists some $\delta>0$ such that whenever $\left\{x_{n}\right\}_{n}$ is a sequence in the closed unit ball of $X$, which is $\tau$-convergent to a point $x \in X$ with $\inf _{n \neq m}\left\|x_{n}-x_{m}\right\|>\epsilon$, then $\|x\|<1-\delta$.

Associated with the $\operatorname{UKK}(\tau)$ property, the following modulus is defined:

$$
P_{X, \tau}(\epsilon)=\inf \left\{1-\|x\|:\left\|x_{n}\right\| \leq 1, \tau-\lim _{n} x_{n}=x, \inf _{n \neq m}\left\|x_{n}-x_{m}\right\|>\epsilon\right\} .
$$

In case that $\tau$ is the weak topology the previous coefficient is known as Partington's modulus, and it is clear that a Banach space has the $\operatorname{UKK}(\tau)$ property if and only if $P_{X, \tau}(\epsilon)>$ 0 for every $\epsilon \in(0,2]$. We denote $P_{X, \tau}\left(1^{-}\right)=\lim _{\epsilon \rightarrow 1^{-}} P_{X, \tau}(\epsilon)$. For $\tau$ a linear topology, it is not difficult to check that

$$
\lim \left\|x_{n}\right\| \leq\left(1-P_{X, \tau}\left(1^{-}\right)\right) \lim _{n, m, n \neq m}\left\|x_{n}-x_{m}\right\|
$$

for every (norm) bounded $\tau$-null sequence $\left\{x_{n}\right\}$ such that $\lim _{n, m, n \neq m}\left\|x_{n}-x_{m}\right\|$ exists. Therefore $X$ verifies the $\tau$-GGLD property whenever $X$ is $\operatorname{UKK}(\tau)$. 


\section{Main results}

Let $C$ be a set and $\left\{B_{s}\right\}_{s \in A}$ be a decreasing net of bounded subsets of $X$. It is clear that

$$
\mathcal{A C}\left(\left\{B_{s}\right\}_{s \in A}, C\right)=\bigcap_{n \in \mathbb{N}}\left\{x \in C: r(x) \leq r_{0}+\frac{1}{n}\right\} .
$$

Therefore, if the set $C$ is $\tau$-sequentially compact and the function $r(\cdot)$ is $\tau$-slsc, the asymptotic center $\mathcal{A C}\left(\left\{B_{s}\right\}_{s \in A}, C\right)$ is a nonempty, $\tau$-sequentially compact set. If $C$ is convex, so is $\mathcal{A C}\left(\left\{B_{s}\right\}_{s \in A}, C\right)$.

We now prove the following technical lemma.

Lemma 3.1 Let $C$ be a convex bounded subset of $X$. Let $\left\{B_{s}\right\}_{s \in A}$ be a decreasing net of subsets of $C$ such that $\mathcal{A C}\left(\left\{B_{s}\right\}_{s \in A}, C\right)=C$. If $C$ is (norm) separable, then there exists $\left\{x_{n}\right\} \subset C$ such that

$$
\lim _{n}\left\|x_{n}-x\right\|=r_{0}
$$

for every $x \in C$, where $r_{0}$ denotes the asymptotic radius of $C$ with respect to the net $\left\{B_{s}\right\}_{s}$.

Proof We will use a similar argument to that of Theorem 2 in [11]. Let $\left\{y_{n}\right\}$ be a dense sequence in $C$ and define $\bar{y}_{n}=\sum_{i=1}^{n} \frac{y_{i}}{n}$.

Since $y_{1} \in C=\mathcal{A C}\left(\left\{B_{s}\right\}_{s}, C\right)$, we can find $s_{1} \in S$ such that

$$
r_{0} \leq r_{s_{1}}\left(y_{1}\right) \leq r_{0}+1
$$

Then select any $x_{1} \in B_{s_{1}}$ such that $\left\|x_{1}-y_{1}\right\| \geq r_{0}-1$.

Assume we have constructed $x_{1}, x_{2}, \ldots, x_{n-1}$ such that for all $1 \leq j \leq k \leq n-1$ we have

$$
r_{0}-\frac{2}{k}+\frac{1}{k^{2}} \leq\left\|x_{k}-y_{j}\right\| \leq r_{0}+\frac{1}{k^{2}} .
$$

Take $s_{n} \in S$ such that $r_{s_{n}}\left(y_{i}\right) \leq r_{0}+\frac{1}{n^{2}}, i=1, \ldots, n$ and $r_{s_{n}}\left(\bar{y}_{n}\right) \leq r_{0}+\frac{1}{n^{2}}$. Select $x_{n} \in B_{s_{n}}$ such that $r_{0}-\frac{1}{n^{2}} \leq\left\|x_{n}-\bar{y}_{n}\right\|$.

Fix $k \leq n$. We then have the following inequalities:

$$
\begin{aligned}
r_{0}-\frac{1}{n^{2}} & \leq\left\|x_{n}-\bar{y}_{n}\right\| \leq \sum_{i=1}^{n} \frac{\left\|x_{n}-y_{i}\right\|}{n} \\
& =\frac{\left\|x_{n}-y_{k}\right\|}{n}+\sum_{i=1, i \neq k}^{n} \frac{\left\|x_{n}-y_{i}\right\|}{n} \\
& \leq \frac{\left\|x_{n}-y_{k}\right\|}{n}+\sum_{i=1, i \neq k}^{n} \frac{r_{0}+\frac{1}{n^{2}}}{n} \\
& =\frac{\left\|x_{n}-y_{k}\right\|}{n}+\left(\frac{n-1}{n}\right)\left(r_{0}+\frac{1}{n^{2}}\right) .
\end{aligned}
$$

From this we obtain that $\frac{r_{0}}{n}-\frac{2}{n^{2}}+\frac{1}{n^{3}} \leq \frac{\left\|x_{n}-y_{k}\right\|}{n}$ and it follows that

$$
r_{0}-\frac{2}{n}+\frac{1}{n^{2}} \leq\left\|x_{n}-y_{k}\right\| \leq r_{s_{n}}\left(y_{k}\right) \leq r_{0}+\frac{1}{n^{2}} .
$$


Thus for a fixed $k$, it easily follows $\lim _{n \rightarrow \infty}\left\|x_{n}-y_{k}\right\|=r_{0}$. Since $\left\{y_{n}\right\}$ is dense in $C$, we deduce $\lim _{n \rightarrow \infty}\left\|x_{n}-x\right\|=r_{0}$ for all $x \in C$.

Recall that every left reversible semitopological semigroup $S$ becomes a directed set when the following partial order is defined:

$$
a, b \in S, \quad a \geq b \quad \Longleftrightarrow \quad a S \subset \operatorname{cl}(b S)
$$

where $\operatorname{cl}(b S)$ denotes the topological closure of the right ideal $b S$.

Let $C$ be subset of $X, S$ be a left reversible semitopological semigroup, and consider a nonexpansive action of $S$ acting on $C$. For a fixed element $u \in C$ define $W_{s}=\operatorname{cl}(s S(u))$, where here the closure is taken for the norm topology. The sets $\left\{W_{s}: s \in S\right\}$ form a nondecreasing family of subsets of $C$. In this case define $r(x)=\lim _{s} r\left(x, W_{s}\right)$. Moreover,

$$
r_{t s}(t x) \leq r_{s}(x)
$$

for all $t, s \in S$ and $x \in C$. Indeed

$$
\begin{aligned}
r_{t s}(t x) & =\sup _{y \in W_{t s}}\|t x-y\|=\sup _{y \in t s S(u)}\|t x-y\|=\sup _{p \in S}\|t x-t s p(u)\| \\
& \leq \sup _{p \in S}\|x-s p(u)\| \leq \sup _{y \in W_{s}}(x)=r_{s}(x) .
\end{aligned}
$$

Therefore $r(t x)=\inf _{s} r_{s}(t x) \leq \inf _{s} r_{s}(x)=r(x)$ for every $t \in S$, and this implies that the set

$$
C(\lambda):=\{x \in C: r(x) \leq \lambda\}
$$

is either empty or $S$-invariant for every $\lambda>0$.

As a consequence, the following lemma is known.

Lemma 3.2 Let $X$ be a Banach space endowed with a topology $\tau$. Let $C$ be a closed convex bounded subset of $X$ which is $\tau$-sequentially compact. Let $S$ be a left reversible semitopological semigroup and consider a nonexpansive action of $S$ on the set $C$. Assume that the function $r(\cdot)$ is $\tau$-slsc. Then

$$
\mathcal{A C}\left(\left\{W_{s}\right\}_{s \in S}, C\right)
$$

is a nonempty closed convex $\tau$-sequentially compact set which is S-invariant.

Next we obtain fixed point results by means of the $\tau$-GGLD and the Opial property.

Theorem 3.3 Let X be a Banach space and $\tau$ be a topology on X. Let C be a (norm) separable closed, convex, bounded, $\tau$-compact and $\tau$-sequentially compact subset of $X$. Let $S$ be a left reversible semigroup generating a nonexpansive action over $C$. Assume that for some $u \in C$ the previous function $r(\cdot)$ is $\tau$-slsc. If $X$ verifies either the $\tau$-GGLD property or the Opial condition with respect to $\tau$, then $\operatorname{Fix}(S) \neq \emptyset$. In case of the weak topology, the separability of $C$ is not necessary. 
Proof Let $\mathcal{F}$ be the family of nonempty, convex, $\tau$-closed and $S$-invariant subsets of $C$. Ordering the family by inclusion and using Zorn's lemma, we obtain a set which is minimal with respect to being nonempty, convex, $\tau$-closed and $S$-invariant. We can then assume that $C$ is the minimal set.

Since $\mathcal{A C}\left(\left\{W_{s}\right\}_{s}, C\right)$ is also a nonempty, convex, $\tau$-closed and $S$-invariant subset of $C$, we have that $\mathcal{A C}\left(\left\{W_{s}\right\}_{s \in S}, C\right)=C$. Let $r_{0}$ denote the asymptotic radius of $C$ with respect to $\left\{W_{s}\right\}_{s \in S}$ and take $\left\{x_{n}\right\}_{n}$ as in Lemma 3.1. By Theorem III.1.5 of [12] we can further assume that $\lim _{n, m, n \neq m}\left\|x_{n}-x_{m}\right\|$ exists and it must then be equal to $r_{0}$. Since $C$ is $\tau$-sequentially compact, we can assume that $\left\{x_{n}\right\}_{n}$ is $\tau$-convergent, say to some $x_{0} \in C$. We have that $\lim _{n}\left\|x_{n}-x_{0}\right\|=r_{0}$, which contradicts the $\tau$-GGLD property since $\tau-\lim _{n}\left(x_{n}-x_{0}\right)=0$. On the other hand, for some $y \in C$ with $y \neq x_{0}$, we obtain

$$
r_{0}=\lim _{n}\left\|x_{n}-x_{0}\right\|=\lim _{n}\left\|x_{n}-x_{0}-\left(y-x_{0}\right)\right\|=r_{0},
$$

which contradicts the Opial condition. Therefore $\operatorname{Fix}(S) \neq \emptyset$.

In case that $\tau$ coincides with the weak topology, it is known that both the $w$-GGLD condition and the Opial condition for the weak topology imply weak normal structure [10] and therefore the $w$-FPP for left reversible semigroups [3].

Notice that for separable Banach spaces and topologies $\tau$ weaker than the norm topology, the $\tau$-compactness of the domain is a superfluous assumption. Indeed, the separability of $X$ implies that $X$ is Lindelöf for the norm and so does for the topology $\tau$ since it is weaker that the norm topology. Thus, $\tau$-sequentially compact sets are countably compact and Lindelöf, so they are $\tau$-compact.

Many examples of Banach spaces are known to satisfy the GGLD condition or the Opial property with respect to some classical topologies. However, to apply Theorem 3.3, the $\tau$-sequential lower semicontinuity of the function $r(\cdot)$ for some $u \in C$ must be checked. In what follows we study equivalent and sufficient conditions to assure this statement.

Let $\left\{x_{n}\right\}$ be a bounded sequence. We define the type function associated to the sequence $\left\{x_{n}\right\}_{n}$ by

$$
\Gamma(x)=\limsup _{n}\left\|x-x_{n}\right\|, \quad x \in X .
$$

In case that $\tau-\lim _{n} x_{n}=0$ we say that $\Gamma$ is a $\tau$-null type function.

Recall that given $\left\{B_{s}\right\}_{s \in A}$ a decreasing net of bounded subsets of $X$ we defined $r(\cdot)$ associated to the net $\left\{B_{s}\right\}_{s \in A}$ as $r(x)=\lim _{s} r\left(x, B_{s}\right)$. The following lemma will be very helpful to assure whether the function $r(\cdot)$ is $\tau$-sequentially lower semicontinuous.

Lemma 3.4 The function $r(\cdot)$ is $\tau$-slsc if and only if the type functions $\Gamma(\cdot)$ are $\tau$-slsc.

Proof One implication is direct since we can take $B_{s}=\left\{x_{n}: n \geq s\right\}$. Then $\Gamma(x)=r(x)=$ $\lim \sup _{n}\left\|x_{n}-x\right\|$.

Assume that the type functions are $\tau$-sequentially lower semicontinuous. Take $\left\{B_{s}\right\}_{s \in A}$ any decreasing net of bounded subsets, and let $\left(y_{n}\right)_{n}$ be a $\tau$-convergent sequence to some point $y \in X$. We have to prove that $r(y) \leq \liminf _{n} r\left(y_{n}\right)$.

Consider a sequence $\left(\epsilon_{n}\right)_{n}$ of positive real numbers with $\lim _{n} \epsilon_{n}=0$. 
We claim that there exists a sequence $\left\{x_{n}\right\}_{n}$ with $x_{n} \in B_{s_{n}}$ and $s_{n}>\cdots>s_{1}$, such that

$$
\begin{aligned}
& r(y)-\epsilon_{n} \leq\left\|y-x_{n}\right\|<r(y)+\epsilon_{n}, \\
& \left\|y_{i}-x_{n}\right\| \leq r\left(y_{i}\right)+\epsilon_{n} ; \quad i=1,2, \ldots n .
\end{aligned}
$$

Indeed, take $s_{1} \in A$ such that

$$
r\left(y, B_{s_{1}}\right)<r(y)+\epsilon_{1} ; \quad r\left(y_{1}, B_{s_{1}}\right)<r\left(y_{1}\right)+\epsilon_{1}
$$

and $x_{1} \in B_{s_{1}}$ with

$$
r(y)-\epsilon_{1}<\left\|y-x_{1}\right\|<r(y)+\epsilon_{1}
$$

Assume now that we have obtained $x_{1}, \ldots, x_{n}$ satisfying (1) and (2). Take $s_{n+1}>s_{n}$ such that

$$
r\left(y, B_{s_{n+1}}\right)<r(y)+\epsilon_{n+1}
$$

and

$$
r\left(y_{i}, B_{s_{n+1}}\right)<r\left(y_{i}\right)+\epsilon_{n+1}
$$

for $i=1, \ldots, n, n+1$. Consider $x_{n+1} \in B_{s_{n+1}}$ with

$$
r(y)-\epsilon_{n+1}<\left\|y-x_{n+1}\right\|<r(y)+\epsilon_{n+1} .
$$

Moreover,

$$
\left\|y_{i}-x_{n+1}\right\| \leq r\left(y_{i}, B_{s_{n+1}}\right) \leq r\left(y_{i}\right)+\epsilon_{n+1} ; \quad i=1,2, \ldots, n+1
$$

and the claim is proved.

By (1), $\lim \sup _{n}\left\|x_{n}-y\right\|=r(y)$ and by (2), $\lim \sup _{n}\left\|x_{n}-y_{i}\right\| \leq r\left(y_{i}\right)$ for every $i \in \mathbb{N}$.

We consider the type function $\Gamma(x)=\limsup _{n}\left\|x_{n}-x\right\|$. We now have

$$
\begin{aligned}
r(y) & =\limsup _{n}\left\|x_{n}-y\right\|=\Gamma(y) \leq \liminf _{i} \Gamma\left(y_{i}\right) \\
& =\liminf _{i} \limsup _{n}\left\|x_{n}-y_{i}\right\| \leq \liminf _{i} r\left(y_{i}\right),
\end{aligned}
$$

which implies that the function $r(\cdot)$ is $\tau$-sequentially lower semicontinuous as we wanted to prove.

In the setting of Theorem 3.3, the set $C$ is $\tau$-sequentially compact, so we can assume that the sequence $\left\{x_{n}\right\}$ obtained in the previous lemma is $\tau$-convergent. Moreover, since the topology is translation invariant, we only need to assume that the $\tau$-null type functions are $\tau$-sequentially lower semicontinuous.

Finally, we can state our main result in this section. 
Theorem 3.5 Let X be a separable Banach space, $\tau$ be a translation invariant topology on $X$ such that $\tau$-compact sets are $\tau$-sequentially compact. Assume that the $\tau$-null functions are $\tau$-slsc. If $X$ verifies either the $\tau$-GGLD property or the $\tau$-Opial condition, $X$ has the $\tau$-FPP for left reversible semigroups.

Notice that when $\tau$ is the weak topology, the separability of $X$ is not necessary.

On the other hand, it is said that the $\tau$-null type functions are constant on spheres if

$$
\limsup _{n}\left\|x_{n}-x\right\|=\limsup _{n}\left\|x_{n}-y\right\|
$$

for every $x, y \in X$ with $\|x\|=\|y\|$, where $\left\{x_{n}\right\}_{n}$ is a norm bounded $\tau$-null sequence. In [11] (Lemma 2) it is proved that the norm and the $\tau$-null type functions are $\tau$-slsc whenever they are constant on spheres.

\section{First examples and applications for the weak-star topology}

In this section we are going to apply Theorem 3.5 to several different classes of dual Banach spaces endowed with their weak* topologies.

To begin with, consider $X=\left(\ell_{1},\|\cdot\|_{1}\right)$, where by $\|\cdot\|_{1}$ we denote the usual norm, and let $\tau$ be the weak* topology $\sigma\left(\ell_{1}, c_{0}\right)$, which is metrizable. It can easily be checked that for every $w^{*}$-null sequence $\left\{x_{n}\right\}_{n}$ and for all $x \in \ell_{1}$,

$$
\limsup \left\|x_{n}+x\right\|_{1}=\|x\|_{1}+\limsup _{n}\left\|x_{n}\right\|_{1}
$$

which implies both the $w^{*}$-GGLD property and the $w^{*}$-sequential lower semicontinuity of the $w^{*}$-null type functions. Therefore we deduce that $\ell_{1}$ has the weak*-FPP for left reversible semigroups [6]. This result can be generalized as follows since the same equality holds for $w^{*}$-null sequences.

Corollary 4.1 Let $\left(X_{n}\right)_{n}$ be a sequence of finite dimensional Banach spaces. Then the onedirect sum

$$
X=\oplus_{1} \sum_{n \in \mathbb{N}} X_{n}
$$

has the weak*-FPP for left reversible semigroups, where the considered predual is defined by $E=\left\{x=\left(x_{n}\right)_{n}: x_{n} \in X_{n}, \lim _{n}\left\|x_{n}\right\|_{X_{n}}=0\right\}$.

In case that $G$ is a separable compact group, its Fourier-Stieltjes algebra $B(G)$ is the direct one-sum of a sequence of finite dimensional Banach spaces (see Section 4 in [13] and Chapter I, Theorem 11.2 in [14]). Applying Corollary 4.1 we can deduce the following.

Corollary 4.2 [5] Let $G$ be a separable compact group and $B(G)$ be its Fourier-Stieltjes algebra. Then $B(G)$ satisfies the $w^{*}$-FPP for left reversible semigroups.

We can now state an improvement of the results in [6] about the $w^{*}$-FPP for left reversible semigroups as follows. 
Theorem 4.3 Let $(X,\|\cdot\|)$ be a Banach space with a boundedly complete Schauder basis $\left\{e_{n}\right\}_{n}$. Assume that for every block basic sequence $\left\{x_{n}\right\}_{n}$ and for every $x \in X$, there holds

$$
\limsup _{n}\left\|x+x_{n}\right\|=\|x\|+\limsup _{n}\left\|x_{n}\right\|
$$

for every $x \in X$. Then $X$ has the $w^{*}$-FPP for left reversible semigroups where $w^{*}=\sigma(X, Z)$ and $Z$ is the closed subspace spanned by the orthogonal functions $\left\{e_{n}^{*}\right\}_{n}$.

Proof When the Schauder basis is boundedly complete, the Banach space $X$ is isomorphic to $Z^{*}$ ([15], Proposition 1.b.4.), and we can consider the weak* topology $\sigma(X, Z)$ where the convergence is equivalent to the pointwise convergence for bounded sequences. Now the $w^{*}$-null type functions are $w^{*}$-sequentially lower semicontinuous since they are constant on spheres and the $w^{*}$-GGLD property is satisfied.

We can apply Theorem 4.3 to more general Banach spaces. The following example is given in [16].

Example 4.4 Let $c_{00}$ be the vector space of all sequences of scalars which are eventually zero. Denote by $[\mathbb{N}]^{<w}$ the subsets of $\mathbb{N}$ with finite cardinality. For $A \subset \mathbb{N}$, let $P_{A}$ be the usual projection, that is, if $x=(x(n))_{n} \in c_{00}, P_{A}(x)$ is the vector whose coordinates are $x(n)$ if $n \in A$ and zero otherwise.

Consider the family $\mathcal{S} \subset[\mathbb{N}]^{<w}$ by

$$
\mathcal{S}:=\left\{S=\left(n_{1}, \ldots, n_{k}\right) \in[\mathbb{N}]^{<w}: n_{i+1} \geq 2 n_{i} \text { for } i=1, \ldots, k-1\right\} .
$$

Define

$$
|x|_{0}:=\sup _{S \in \mathcal{S}}\left\|P_{S}(x)\right\|_{1}
$$

and let $X$ be the completion of $c_{00}$ with the norm $|\cdot|_{0}$. Then $\left(X,|\cdot|_{0}\right)$ is a dual Banach space satisfying the hypotheses of Theorem 4.3. Indeed, the sequence $\left\{e_{n}\right\}$ forms a monotonous bounded complete Schauder basis, which implies that $X$ is isometric to the dual of $Z$, where $Z$ is the Banach space spanned by the orthogonal functionals $\left\{e_{n}^{*}\right\}$. The fact that $X$ satisfies the equality given in Theorem 4.3 is proved in [16]. Therefore, it satisfies the $w^{*}$-FPP for left reversible semigroups. Notice that this Banach space is not isomorphic to $\ell_{1}[16]$.

Corollary 4.5 [7] Let $H$ be a Hilbert separable Banach space. Then $\mathcal{T}(H)$, the space of the trace class operators on $H$, has the weak ${ }^{*}$-FPP for left reversible groups, where the predual is $E=K(H)$, the space of all compact operators defined on $H$.

In this case, Lennard proved that $\mathcal{T}(H)$ verifies the weak* uniform Kadec-Klee property [17] and therefore the $w^{*}$-GGLD condition. Proposition 2.4 in [7] implies that the $w^{*}$-null type functions are $w^{*}$-sequentially lower semicontinuous. (Notice that in [7] the separability of the Hilbert space can be dropped.)

Corollary 4.6 [8] The Hardy space $H^{1}(\Delta)$ has the weak ${ }^{*}$-FPP for left reversible semigroups. 
Notice that $H^{1}(\Delta)$ is a separable Banach space, it has the $w^{*}$-GGLD property since it verifies the $w^{*}$-UKK condition [18], and Lemma 3.2 in [8] implies that the $w^{*}$-null type functions are $w^{*}$-sequentially lower semicontinuous. Here, the weak* topology refers to the isometric predual $C(\mathbb{T}) / A_{0}(\mathbb{T})$, where $C(\mathbb{T})$ is the space of all continuous functions on the torus $\mathbb{T}$, with the usual supremum norm, and $A_{0}(\mathbb{T})$ is the set of boundary values of the disc algebra with zero constant term.

Recall that map $\phi:[0,+\infty) \rightarrow[0,+\infty)$ is said to be an Orlicz function if $\phi$ is convex, vanishing at 0 , continuous and not identically equal to zero. A sequence $\Phi:=\left\{\phi_{n}\right\}_{n}$ of Orlicz functions is called a Musielak-Orlicz function.

Given a Musielak-Orlicz function, a convex modular $I_{\Phi}$ is defined on the set of all real sequences given by

$$
I_{\Phi}(x)=\sum_{n=1}^{\infty} \phi_{n}(|x(n)|) .
$$

A Musielak-Orlicz sequence space generated by $\Phi$ is defined by

$$
\ell_{\Phi}=\left\{x=\left(x_{n}\right): I_{\Phi}(\lambda x)<+\infty \text { for some } \lambda>0\right\} .
$$

We can consider $\ell_{\Phi}$ equipped with the Luxemburg norm

$$
\|x\|:=\inf \left\{k>0: I_{\Phi}(x / k) \leq 1\right\}
$$

or with the Orlicz norm

$$
\|x\|^{o}:=\inf \left\{\frac{1}{k}\left(1+I_{\Phi}(k x)\right): k>0\right\} .
$$

It is well known that both norms are equivalent and $\ell_{\Phi}$ is a Banach space $[19,20]$.

In case that $\phi_{m}=\phi_{n}$ for all $n, m \in \mathbb{N}$, we simply say that $\ell_{\Phi}$ is an Orlicz sequence Banach space.

It is said that a Musielak-Orlicz function $\Phi=\left\{\phi_{n}\right\}_{n}$ satisfies the condition $\delta_{2}$ if there are positive constants $a$ and $K$ and a nonnegative sequence $\left(c_{n}\right) \in \ell_{1}$ such that

$$
\phi_{n}(2 t) \leq K \phi_{n}(t)+c_{n}
$$

for every $n \in \mathbb{N}, t \in \mathbb{R}_{+}$satisfying $\phi_{n}(t) \leq a$.

For Orlicz sequence Banach spaces, it is said that an Orlicz function $\phi$ satisfies the condition $\delta_{2}$ if there exist some $t_{0}>0$ and $K>0$ such that $\phi(2 t) \leq K \phi(t)$ for every $t \in\left[0, t_{0}\right]$.

When the condition $\delta_{2}$ is satisfied, the unit vectors form a boundedly complete normalized unconditional basis of $\ell_{\Phi}$ ([15], Proposition 4.d.3). We denote by $e_{n}^{*}$ the functional vector associated with $e_{n}$ for every $n \in \mathbb{N}$ and consider $\left[e_{n}^{*}\right]$ the closed span of the vectors $\left\{e_{n}^{*}\right\}$ in the dual of the Musielak-Orlicz space. Since the Schauder basis is monotone, the dual of the Banach space $\left[e_{n}^{*}\right]$ is isometric to $\ell_{\phi}$ ([15], Proposition 1.b.4.). Therefore we can consider the weak* topology $\sigma\left(\ell_{\Phi},\left[e_{n}^{*}\right]\right)$. At this point we can state the following.

Theorem 4.7 Let $\Phi=\left\{\phi_{n}\right\}$ be a Musielak-Orlicz function satisfying the condition $\delta_{2}$. The Musielak-Orlicz space satisfies the $w^{*}$-FPP for left reversible semigroups for both the Luxemburg and the Orlicz norm. 
Proof Notice that $\ell_{\Phi}$ is a separable Banach space. The arguments included in the proof of (iii) $\Rightarrow$ (i) of Theorem 1 in [21] imply that $\ell_{\Phi}$ endowed with the Luxemburg norm satisfies the $w^{*}$-uniform Opial condition. From the proof of Theorem 2 in [22] we can deduce that the Musielak-Orlicz space $\ell_{\Phi}$ satisfies the $w^{*}$-uniform Kadec-Klee property when it is equipped with the Orlicz norm and so the $w^{*}$-GGLD property. Since the weak* convergence implies the coordinatewise convergence, standard arguments show that

$$
\limsup _{n} I_{\Phi}\left(x_{n}+x\right)=\limsup _{n} I_{\Phi}\left(x_{n}\right)+I_{\Phi}(x)
$$

for every $x \in \ell_{\Phi}$ and for every $w^{*}$-null sequence $\left\{x_{n}\right\}$. This implies that the functional $x \in$ $\ell_{\Phi} \rightarrow \lim \sup _{n} I_{\phi}\left(x_{n}-x\right)$ is $w^{*}$-slsc. With this property, it is easy to check the $w^{*}$-sequential lower semicontinuity for the $w^{*}$-null type functions for both norms, the Luxemburg and the Orlicz norm.

Particular examples of Musielak-Orlicz sequence spaces are the Nakano spaces $\ell^{\left(p_{n}\right)}$ where the Orlicz functions are $\phi_{n}(t)=|t|^{p_{n}}$ with $p_{n} \subset[1,+\infty)$ [23]. In this case, the Musielak-Orlicz function satisfies the condition $\delta_{2}$ if and only if $\sup _{n} p_{n}<+\infty$. In case that $p_{n}=1$ for every $n \in \mathbb{N}$, we obtain $\ell_{1}$. However, there exist some dual Nakano spaces which are not isomorphic to $\ell_{1}$ and satisfying $\lim _{n} p_{n}=1$ [23].

Corollary 4.8 Let $\left(p_{n}\right)$ be a bounded sequence in $[1,+\infty)$. Then the corresponding Nakano sequence Banach space verifies the $w^{*}$-FPP for left reversible semigroups.

In the rest of this section we will consider some relevant equivalent norms in $\ell_{1}$, and we will study whether they provide the $w^{*}$-FPP for left reversible semigroups. On the one hand, there are some equivalent norms in $\ell_{1}$ which fail to have this property. For instance $\ell_{1}$ endowed with the norm $\|x\|=\max \left\{\left\|x^{+}\right\|_{1},\left\|x^{-}\right\|_{1}\right\}$, as the dual of $c_{0}$ with the norm $\left\|x^{+}\right\|_{\infty}+\left\|x^{-}\right\|_{\infty}$, fails the $w^{*}$-FPP for nonexpansive mappings [6]. On the other hand, the $\tau$-GGLD and the Opial conditions are properties that can be transferred by isomorphisms under certain conditions. For instance, it is known (see [11]) that if we consider $Y=\left(\ell_{1},|\cdot|\right)$ for some equivalent norm, then $Y$ satisfies the $\sigma\left(\ell_{1}, c_{0}\right)$-GGLD condition whenever $d\left(Y,\left(\ell_{1},\|\cdot\|_{1}\right)\right)<2$ (and this is the best upper bound that can be obtained due to the previous norm). However, as far as we know, the $w^{*}$-FPP for left reversible semigroups cannot be derived from stability results since the same does not hold for the $\tau$-sequential lower semicontinuity of the $\tau$-null type functions. Indeed, consider the Euclidean norm $\|\cdot\|_{2}$ in $\mathbb{R}^{2}$ and define the equivalent norm in $\ell_{1}$ by

$$
v(x):=\left\||x(1)| u_{1}+\sum_{n=2}^{\infty}|x(n)| u_{2}\right\|_{2},
$$

where $u_{1}=(1,0)$ and $u_{2}=\left(-a,\left(1-a^{2}\right)^{1 / 2}\right)$ for some $a \in(0,1)$. Finally, for $\lambda>0$, define the norm

$$
|x|_{\lambda}:=\|x\|_{1}+\lambda v(x)
$$

for all $x \in \ell_{1}$. It is not difficult to check that $|\cdot|_{\lambda}$ is equivalent to $\|\cdot\|_{1}$ and that the BanachMazur distance between $\left(\ell_{1},\|\cdot\|_{1}\right)$ and $\left(\ell_{1},|\cdot|_{\lambda}\right)$ tends to one when $\lambda$ goes to zero. Consider 
$x_{n}=e_{1}+a e_{n}$, which tends to $x=e_{1}$ in the $\sigma\left(\ell_{1}, c_{0}\right)$-topology. Notice that

$$
|x|_{\lambda}=1+\lambda>1+\lambda\left(1-a^{2}\right)^{\frac{1}{2}}=\left|x_{n}\right|_{\lambda}
$$

for every $n \in \mathbb{N}$. Hence the norm $|\cdot|_{\lambda}$ is not $\sigma\left(\ell_{1}, c_{0}\right)$-sequentially lower semicontinuous, and consequently $\sigma\left(\ell_{1}, c_{0}\right)$-null type functions are not $\sigma\left(\ell_{1}, c_{0}\right)$-slsc in general.

A relevant renorming in metric fixed point theory was given by Lin in [24] in the following way: Let $\left\{\gamma_{k}\right\}$ be a nondecreasing sequence of positive numbers converging to 1 and define

$$
\left\|\left\{a_{n}\right\}\right\|:=\sup _{k} \gamma_{k} \sum_{n=k}^{\infty}\left|a_{n}\right|
$$

for every $\left\{a_{n}\right\} \in \ell_{1}$. Lin proved that $\ell_{1}$ endowed with this norm verifies the fixed point property for nonexpansive mappings, that is, every $\||\cdot|||$-nonexpansive mapping defined on a closed convex bounded subset of $\ell_{1}$ into itself has a fixed point. This assertion proves that FPP does not imply reflexivity as it was conjectured for a long time.

At this point, we do not know whether $\left(\ell_{1},\||\cdot|\|\right)$ verifies a similar statement for left reversible semigroups of nonexpansive mappings. Here we prove that $\left(\ell_{1},\||\|\mid\|)\right.$ does verify the $w^{*}$-FPP for left reversible semigroups where by the weak ${ }^{*}$ topology we refer to $\sigma\left(\ell_{1}, c_{0}\right)$. Notice that the above norm is a dual norm, that is, if $X=\left(\ell_{1},\|\mid\| \|\right)$ then $X$ is isometric to a dual space. This can be deduced from the fact that the Schauder basis $\left\{e_{n}\right\}$ is boundedly complete and it is monotonous for the $\|\cdot\| \|$ norm. Moreover, $X$ is isometric to the dual of the Banach space spanned by $\left[e_{n}^{*}\right]$ so the weak* topology is in fact $\sigma\left(\ell_{1}, c_{0}\right)$ (see Proposition 1.b.4 in [15]).

If $\left\{x_{n}\right\}_{n}$ is a $w^{*}$-null sequence in $\ell_{1}$, then $\lim \sup _{n}\left\|x_{n}-x_{m}\right\|\left|=2 \lim \sup _{n}\left\|x_{n}\right\|\right|[24]$, which clearly implies the $w^{*}$-GGLD. Notice that the $w^{*}$-null type functions are not constant in spheres: consider the sequence $x_{n}=e_{n}$ for every $n \in \mathbb{N}$ and $x=\frac{1}{\gamma_{1}} e_{1}, y=\frac{1}{\gamma_{2}} e_{2}$. Now $\|x\|=$ $\|y\|=1$ but

$$
\underset{n}{\limsup }\left\|\mid x_{n}-x\right\|=1+\gamma_{1} \quad \text { and } \quad \limsup _{n}\left\|x_{n}-x\right\| \|=1+\gamma_{2}
$$

so we cannot derive the $w^{*}$-sequential lower semicontinuity of the $w^{*}$-null type functions from this fact. We will consider the following result which is due to Lennard and it was included in [25]. However, we here develop a shorter proof for the sake of completeness.

Lemma 4.9 Let $\left\{x_{n}\right\}_{n}$ be a $w^{*}$-null sequence in $\ell_{1}$. Define

$$
\Gamma(y):=\limsup _{n \rightarrow \infty}\left\|x_{n}-y\right\| .
$$

Then

$$
\Gamma(y)=\sup _{k \in \mathbb{N}}\left\{\gamma_{k}\left(\sum_{n=k}^{\infty}\left|y_{n}\right|+\underset{n}{\limsup \|}\left\|x_{n}\right\| \|\right)\right\}
$$

for every $y \in \ell_{1}$ with $y=\sum_{n=1}^{\infty} y_{n} e_{n}$. 
Proof We may, without loss of generality, assume that the sequence $\left\{x_{n}\right\}$ is disjointly supported.

First of all, assume that the vector $y$ is finitely supported, that is, there exists some $n_{0} \in \mathbb{N}$ such that $y_{n}=0$ if $n>n_{0}$. We can assume that $n_{0}<\min \left\{\operatorname{supp}\left(x_{1}\right)\right\}$. In such case, for every $n \in \mathbb{N}$,

$$
\left\|y-x_{n}\right\| \mid=\max \left\{\sup _{1 \leq k \leq n_{0}} \gamma_{k}\left(\sum_{n=k}^{n_{0}}\left|y_{n}\right|+\left\|x_{n}\right\|_{1}\right),\left\|x_{n}\right\| \mid\right\} .
$$

Taking limits when $n$ goes to infinity and using that $\limsup _{n}\left\|x_{n}\right\|_{1}=\lim \sup _{n}\|\| x_{n} \|||$ since $\left\{x_{n}\right\}$ is disjointly supported, we deduce

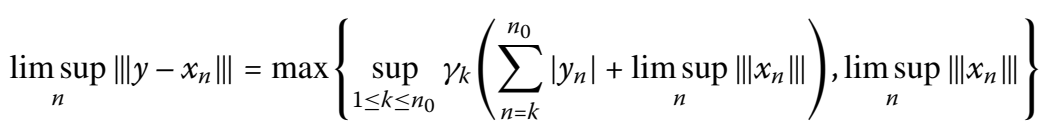

$$
\begin{aligned}
& =\sup _{k \in \mathbb{N}}\left\{\gamma_{k}\left(\sum_{n=k}^{\infty}\left|y_{n}\right|+\limsup _{n}\left\|\left|x_{n} \|\right|\right)\right\}\right. \text {, }
\end{aligned}
$$

where the last equality follows from the fact that $\lim _{k} \gamma_{k}=1$ and $y_{n}=0$ if $n>n_{0}$. Then the lemma holds in this case.

Let $y=\sum_{n=1}^{\infty} y_{n} e_{n}$ be any vector in $\ell_{1}$ and denote $y_{s}=\sum_{n=1}^{s} y_{n} e_{n}$. Notice that $\Gamma$ is a continuous function for the norm topology so $\Gamma(y)=\lim _{s} \Gamma\left(y_{s}\right)$. If we prove that $\lim _{s} \Gamma\left(y_{s}\right)$ coincides with the right part of the equality stated in the lemma, the proof will be finished.

For every $s \in \mathbb{N}$,

$$
\begin{aligned}
& \left.\Gamma\left(y_{s}\right)=\max \left\{\sup _{1 \leq k \leq s} \gamma_{k}\left(\sum_{n=k}^{s}\left|y_{n}\right|+\underset{n}{\lim \sup }\left\|\left|x_{n}\right|\right\|\right), \underset{n}{\limsup }\left\|x_{n}\right\|\right\}\right\} \\
& \leq \max \left\{\sup _{k \geq 1} \gamma_{k}\left(\sum_{n=k}^{\infty}\left|y_{n}\right|+\underset{n}{\lim \sup }\left\|x_{n}\right\| \mid\right), \underset{n}{\limsup }\left\|\left|x_{n} \|\right|\right\}\right. \\
& =\sup _{k \in \mathbb{N}}\left\{\gamma_{k}\left(\sum_{n=k}^{\infty}\left|y_{n}\right|+\limsup _{n}\left\|x_{n}\right\|\right)\right\} \text {. }
\end{aligned}
$$

On the other hand, let $\epsilon>0$ and $s_{0} \in \mathbb{N}$ such that $\left\|y-y_{s}\right\|_{1}<\epsilon$ for every $s \geq s_{0}$. Fix $s \geq s_{0}$. In this case

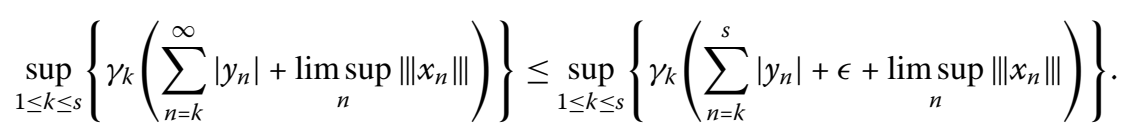

And

$$
\begin{aligned}
& \sup _{s<k}\left\{\gamma_{k}\left(\sum_{n=k}^{\infty}\left|y_{n}\right|+\limsup _{n}\left\|x_{n}\right\| \mid\right)\right\} \\
& \leq \sup _{s<k}\left\{\gamma_{k}\left(\epsilon+\underset{n}{\limsup }\|\| x_{n}\|\|\right)\right\}=\epsilon+\underset{n}{\lim \sup }\|\| x_{n}\|\| .
\end{aligned}
$$


In any case,

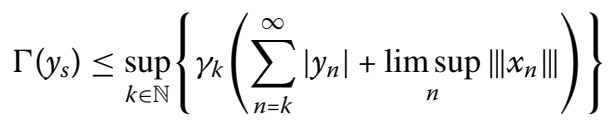

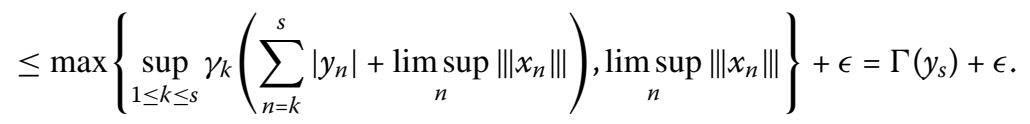

Taking limits when $s$ goes to infinity and having in mind that $\epsilon$ is arbitrary, we obtain the desired equality.

By using the previous equality we can now check the following.

Lemma 4.10 For the space $\left(\ell_{1},|| \cdot \mid \|\right), w^{*}$-null type functions are $w^{*}$-sequentially semicontinuous.

Proof Let $\left\{y_{m}\right\}$ be a $w^{*}$ convergent sequence and take $y$ its $w^{*}$ limit.

Take $P_{k}$ the natural projections associated to the usual basis of $\ell_{1}$ and take $Q_{n}=I-P_{n}$. Fix $n \in \mathbb{N}$. By Lemma 4.9, we have that $\Gamma\left(y_{s}\right)=\sup _{k \in \mathbb{N}}\left\{\gamma_{k}\left(\Gamma(0)+\left\|Q_{k-1}\left(y_{s}\right)\right\|_{1}\right)\right\} \geq \gamma_{n}(\Gamma(0)+$ $\left.\left\|Q_{n-1}\left(y_{s}\right)\right\|_{1}\right)$.

It is clear that $Q_{n-1}\left(y_{k}\right)$ converge $w^{*}$ to $Q_{n-1}(y)$. Using that $\|\cdot\|_{1}$ is a $w^{*}$ lower semicontinuous function, we obtain that

$$
\begin{aligned}
\liminf _{m \rightarrow \infty} \Gamma\left(y_{m}\right) & \geq \liminf _{m \rightarrow \infty} \gamma_{n}\left(\Gamma(0)+\left\|Q_{n-1}\left(y_{m}\right)\right\|_{1}\right) \\
& =\gamma_{n} \Gamma(0)+\gamma_{n} \liminf _{m \rightarrow \infty}\left\|Q_{n-1}\left(y_{m}\right)\right\|_{1} \\
& \geq \gamma_{n} \Gamma(0)+\gamma_{n}\left\|Q_{n-1}(y)\right\|_{1} .
\end{aligned}
$$

This inequality then holds for each $n \in \mathbb{N}$, which proves that $\liminf _{m \rightarrow \infty} \Gamma\left(y_{m}\right) \geq$ $\sup _{n \in \mathbb{N}}\left\{\gamma_{n}\left(\Gamma(0)+\left\|Q_{n-1}(y)\right\|_{1}\right)\right\}=\Gamma(y)$ as we wanted to prove.

Using Theorem 3.5 we finally deduce the following.

Corollary 4.11 $\left(\ell_{1}, \||\cdot| \mid\right)$ has the $w^{*}$-FPP for left reversible semigroups.

Example 4.12 Consider $X=\ell_{1}$ endowed with the equivalent norm

$$
|x|=\max \left\{\frac{1}{4}\|x\|_{1}, \max _{n}\{|x(n)|\}+\sum_{n=1}^{\infty} \frac{|x(n)|}{2^{n}}\right\} .
$$

Consider $\tau$ as the weak ${ }^{*}$ topology $\sigma\left(\ell_{1}, c_{0}\right)$. Notice that $\left(\ell_{1},|\cdot|\right)$ is a dual space since the Schauder basis $\left\{e_{n}\right\}$ is boundedly complete and monotonous. Taking the sequence $x_{n}=e_{n}$ for $n \in \mathbb{N}$, we obtain $\lim _{n}\left\|x_{n}\right\|=1=\lim _{n, m ; n \neq m}\left\|x_{n}-x_{m}\right\|=1$, which implies that $X$ fails the $w^{*}$-GGLD condition. However, it is easy to check that $X$ has the Opial condition with respect to the $\sigma\left(\ell_{1}, c_{0}\right)$ topology and that the $\sigma\left(\ell_{1}, c_{0}\right)$-null type functions are sequentially lower semicontinuous. From Theorem 3.5 we can deduce the $w^{*}$-FPP for left reversible semigroups in the space $\left(\ell_{1},|\cdot|\right)$. 
We finish the section with the Banach space introduced in Example 2.1, for which we showed that the asymptotic centers were not weakly compact in general, so the arguments used in $[5,6,8]$ or $[7]$ are not valid for proving the $w^{*}$-FPP for left reversible semigroups.

Example 4.13 Let $X:=\left(\ell_{1},\|\cdot\|\right)$, where the norm $\|\cdot\|$ is defined as

$$
\|x\|=\max _{n \in \mathbb{N}}\left(|x(n)|+\frac{1}{2} \sum_{i=n+1}^{\infty}|x(i)|\right) .
$$

Here $\frac{1}{2}\|x\|_{1} \leq\|x\| \leq \frac{3}{2}\|x\|_{1}$ for every $x \in \ell_{1}$. Notice that if $x, y$ are vectors in $\ell_{1}$ such that $\max \{\operatorname{supp}(x)\}<\min \{\operatorname{supp}(y)\}$, then it is not difficult to check that

$$
\|x+y\|=\max \left\{\|y\|,\|x\|+\frac{1}{2}\|y\|_{1}\right\} .
$$

The previous equality also proves that the Schauder basis is monotonous for $\|\cdot\|$ and $X$ is isometric to a dual space. Assume that $\left\{x_{n}\right\}$ is a weak*-null sequence, where by $w^{*}$ topology we mean the $\sigma\left(\ell_{1}, c_{0}\right)$ topology. Without loss of generality, we can assume that $\left\{x_{n}\right\}$ is a block basic sequence, $l=\lim _{n}\left\|x_{n}\right\|$ exists and that $\lim _{n, m ; n \neq m}\left\|x_{n}-x_{m}\right\|=$ $\lim \sup _{n} \lim \sup _{m}\left\|x_{n}-x_{m}\right\|$. Therefore

$$
\begin{aligned}
& \underset{n}{\limsup } \limsup _{m}\left\|x_{n}-x_{m}\right\|=\underset{n}{\limsup \lim \sup \max }\left\{\left\|x_{m}\right\|,\left\|x_{n}\right\|+\frac{1}{2}\left\|x_{m}\right\|_{1}\right\} \\
& =\underset{n}{\lim \sup \max }\left\{l,\left\|x_{n}\right\|+\frac{1}{2} \underset{m}{\limsup }\left\|x_{m}\right\|_{1}\right\} \\
& =l+\frac{1}{2} \limsup _{m}\left\|x_{m}\right\|_{1} \geq \frac{4}{3} l,
\end{aligned}
$$

which implies the $w^{*}$-GGLD property.

Let us prove that the weak* ${ }^{*}$-null type functions are $w^{*}$-lower semicontinuous.

Take $\left\{x_{n}\right\}$ a $w^{*}$-null sequence and $y=\sum_{n=1}^{\infty} y_{n} e_{n} \in \ell_{1}$. We can assume that $\left\{x_{n}\right\}$ is a block basic sequence. Let $\epsilon>0$ and $s_{0} \in \mathbb{N}$ such that $\left\|y-y_{s}\right\|<\epsilon$ for every $s \geq s_{0}$, where $y_{s}=$ $\sum_{n=1}^{s} y_{n} e_{n}$. Notice that $\Gamma(y)=\lim \sup _{n}\left\|y-x_{n}\right\|=\lim _{s} \lim _{\sup _{n}}\left\|y_{s}-x_{n}\right\|$. Moreover, from the definition of the norm and from the equality (4),

$$
\begin{aligned}
\underset{n}{\limsup \left\|y_{s}-x_{n}\right\|} & =\max \left\{\limsup _{n}\left\|x_{n}\right\|,\left\|y_{s}\right\|+\frac{1}{2} \limsup _{n}\left\|x_{n}\right\|_{1}\right\} \\
& \leq \max \left\{\limsup _{n}\left\|x_{n}\right\|,\|y\|+\frac{1}{2} \limsup _{n}\left\|x_{n}\right\|_{1}\right\}
\end{aligned}
$$

for every $s \in \mathbb{N}$. On the other hand, for every $s \geq s_{0}$,

$$
\begin{aligned}
& \max \left\{\limsup _{n}\left\|x_{n}\right\|,\|y\|+\frac{1}{2} \limsup _{n}\left\|x_{n}\right\|_{1}\right\} \\
& \leq \max \left\{\underset{n}{\limsup }\left\|x_{n}\right\|,\left\|y_{s}\right\|+\frac{1}{2} \underset{n}{\limsup }\left\|x_{n}\right\|_{1}\right\}+\epsilon \\
& =\underset{n}{\limsup }\left\|y_{s}-x_{n}\right\|+\epsilon \text {. }
\end{aligned}
$$


Taking limits when $s$ goes to infinity, we deduce that for every $y \in \ell_{1}$,

$$
\underset{n}{\limsup }\left\|y-x_{n}\right\|=\max \left\{\limsup _{n}\left\|x_{n}\right\|,\|y\|+\frac{1}{2} \limsup _{n}\left\|x_{n}\right\|_{1}\right\}
$$

The above implies that the $w^{*}$-null type functions are constant on spheres and therefore $w^{*}$-slsc.

Therefore $\left(\ell_{1},\|\cdot\|\right)$ verifies the $w^{*}$-FPP for left reversible semigroups Notice also that this space fails the Opial condition with respect to the $w^{*}$ topology. Indeed, consider the weak*-null sequence $x_{n}=e_{n}$ for $n \in \mathbb{N}$ and the vector $x=\frac{1}{2} e_{1}$. Then $\lim _{n}\left\|x_{n}\right\|=$ $\lim _{n}\left\|x_{n}+x\right\|$.

\section{Modular function spaces and the $\boldsymbol{\rho}$-FPP for left reversible semigroups}

In this section we consider modular function spaces $L_{\rho}:=\{f \in \mathcal{M}: \rho(\alpha f) \rightarrow 0$ as $\alpha \rightarrow 0\}$ endowed with the Luxemburg norm

$$
\|f\|:=\inf \left\{\alpha>0: \rho\left(\frac{f}{\alpha}\right) \leq 1\right\}
$$

and the Orlicz norm

$$
\|f\|_{o}:=\inf \left\{\frac{1}{k}(1+\rho(k f)): k>0\right\}
$$

Here $\mathcal{M}$ denotes a set of measurable functions and $\rho$ a convex additive function modular defined over $\mathcal{M}$. It is said that a sequence $\left(f_{n}\right) \subset L_{\rho}$ converges to $f \rho$-almost everywhere, $f_{n} \rightarrow f \rho$-a.e., if $\left\{w \in \Omega: f(w) \neq \lim _{n} f_{n}(w)\right\}$ is $\rho$-null. For all general definitions we refer to $[20,26]$ or $[27]$.

A function modular $\rho$ is said to satisfy the $\Delta_{2}$-type condition if there exists some $K>0$ such that $\rho(2 f) \leq K \rho(f)$ for every $f \in L_{\rho}$.

In this section we assume that $\rho$ is a $\sigma$-finite convex additive function modular which satisfies the $\Delta_{2}$-type condition (see [27] or [28]). In this case, a topology $\tau_{\rho}$ is defined over $L_{\rho}$ such that $\tau_{\rho}$-compact sets coincide exactly with $\tau_{\rho}$-sequentially compact sets [28]. Moreover, the $\rho$-convergence coincides with the $\tau$-convergence up to subsequences.

In [27] (Section 4) it is proved that, under the $\Delta_{2}$-type condition, the modular function space $L_{\rho}$ verifies the $\tau_{\rho}$-uniform Opial condition for both the Luxemburg and the Orlicz norm. Moreover, Lemma 5.3 and Lemma 5.4 of [27] show that the $\tau_{\rho}$-null type functions are $\tau_{\rho}$-sequentially lower semicontinuous. Hence we can state the following theorem.

Theorem 5.1 Let $\rho$ be a $\sigma$-finite convex additive function modular which satisfies the $\Delta_{2}$-condition. Then the modular function space $L_{\rho}$ verifies the $\tau_{\rho}$-FPP for left reversible semigroups when it is equipped with either the Luxemburg or the Orlicz norm.

Examples of modular function spaces are the $L_{p}$-spaces and more generally the Musielak-Orlicz function spaces, where the $\tau_{\rho}$ topology coincides with the local convergence in measure topology. For the definition of Musielak-Orlicz function spaces and for more examples of modular function spaces, see [20] and [26]. 


\section{L-Embedded Banach spaces and the FPP for left reversible semigroups with respect to the abstract measure topology}

In this section we consider $L$-embedded Banach spaces endowed with the so-called measure topology. A Banach space $X$ is said to be an $L$-embedded Banach space if there exists a closed subspace $X_{s} \subset X^{* *}$ such that $X^{* *}=X \oplus_{1} X_{s}$. A wide study and many examples of this class of Banach spaces can be found in the monograph [29]. Examples of $L$-embedded Banach spaces are the following:

(1) Duals of $M$-embedded Banach spaces.

(2) $L_{1}(\mu)$-spaces and preduals of von Neumann algebras.

Recall that a sequence $\left\{x_{n}\right\}$ is said to span an asymptotically isometric copy of $\ell_{1}$ if there exists a nonincreasing sequence $\left\{\delta_{n}\right\} \subset[0,1)$ tending to 0 such that

$$
\sum_{n=1}^{\infty}\left(1-\delta_{n}\right)\left|\alpha_{n}\right| \leq\left\|\sum_{n=1}^{\infty} \alpha_{n} x_{n}\right\| \leq \sum_{n=1}^{\infty}\left|\alpha_{n}\right|
$$

for every $\left\{\alpha_{n}\right\} \in \ell_{1}$. In this case we will denote $x_{n} \sim($ asy $) \ell_{1}$.

For $L$-embedded Banach spaces, the abstract measure topology $\left(\tau_{\mu}\right)$ is defined in [30] (Section 5) by considering the class of convergent sequences. Namely, if $\left\{x_{n}\right\}$ is a sequence in an $L$-embedded Banach space, we say that $\left\{x_{n}\right\}$ tends to 0 in the abstract measure topology $\left(\tau_{\mu}-\lim _{n} x_{n}=0\right)$ if

$\left\{x_{n}\right\}$ is norm bounded and every subsequence $\left\{x_{n_{k}}\right\}$ contains a subsequence $\left\{x_{n_{k}}\right\}$

such that $x_{n_{k_{l}}} /\left\|x_{n_{k_{l}}}\right\| \sim($ asy $) \ell_{1}$ or $\left\|x_{n_{k_{l}}}\right\| \rightarrow 0$.

When $X$ is a separable $L$-embedded Banach space, the notions of compactness and sequential compactness agree for $\tau_{\mu}$ [31].

It is proved in [32] (see also [33]) that for every $\tau_{\mu}$-null sequence $\left\{x_{n}\right\}$ in an $L$-embedded Banach space,

$$
\limsup _{n}\left\|x_{n}+x\right\|=\limsup _{n}\left\|x_{n}\right\|+\|x\|
$$

for all $x \in X$. This equality implies the $\tau_{\mu}$-GGLD property and the $\tau_{\mu}$-sequential lower semicontinuity of the $\tau_{\mu}$-null type functions.

It is known that $L$-embedded Banach spaces satisfy the FPP for nonexpansive mappings with respect to the abstract measure topology [32]. According to Theorem 3.5, we can extend this result to left reversible semigroups in the following way.

Corollary 6.1 Let $X$ be a separable L-embedded Banach space. Then $X$ verifies the $\tau_{\mu}$-FPP for left reversible semigroups.

As a particular case we can deduce Theorem 5.1 in [7] when the Hilbert space $H$ is separable. Indeed, in case that the $L$-embedded Banach space is $L^{1}(\mathcal{M}, \tau)$ for some finite von Neumann algebra $\mathcal{M}$ defined over a Hilbert space, the previous measure topology coincides with the usual measure topology defined on $L^{1}(\mathcal{M}, \tau)$ for bounded sets (see Theorem 1.1 in [31]). Hence, noncommutative $L_{1}$-spaces verify the fixed point property for left reversible semigroups with respect to the usual measure topology. This topology is in fact the convergence locally in measure topology in case that $L^{1}(\mathcal{M}, \tau)=L^{1}(\mu)$ for some $\sigma$-finite measure space. 


\section{Competing interests}

The authors declare that they have no competing interests.

\section{Authors' contributions}

Both authors have contributed equally and significantly in writing this article. Both authors read and approved the final manuscript.

\section{Author details}

${ }^{1}$ CIMAT, Centro de Investigación en Matemáticas, CONACYT, Consejo Nacional de Ciencias y Tecnología, Guanajuato, México. ${ }^{2}$ Departamento de Análisis Matemático, Facultad de Matemáticas, Universidad de Sevilla, Tarfia s/n, Sevilla, 41012, Spain

\section{Acknowledgements}

The authors would like to thank the referees for their valuable suggestions to improve the presentation of this article. The second author is partially supported by MCIN, Grant MTM-2012-34847-C02-01 and Junta de Andalucía, Grants FQM-127 and P08-FQM-03543.

\section{Received: 27 January 2015 Accepted: 18 June 2015 Published online: 09 July 2015}

\section{References}

1. Paterson, ALT: Amenability. Mathematical Surveys and Monographs, vol. 19. Am. Math. Soc., Providence (1988)

2. Alspach, DE: A fixed point free nonexpansive map. Proc. Am. Math. Soc. 82, 423-424 (1981)

3. Lim, T-C: Characterizations of normal structure. Proc. Am. Math. Soc. 34(2), 313-319 (1974)

4. Holmes, RD, Lau, AT: Non-expansive actions of topological semigroups and fixed points. J. Lond. Math. Soc. (2) 5 , 330-336 (1972)

5. Lau, AT-M, Mah, PF: Fixed point property for Banach algebras associated to locally compact groups. J. Funct. Anal. 258, 357-372 (2010)

6. Lim, T-C: Asymptotic centers and nonexpansive mappings in conjugate Banach spaces. Pac. J. Math. 90(1), 135-143 (1980)

7. Randrianantoanina, N: Fixed point properties of semigroups of nonexpansive mappings. J. Funct. Anal. 258, 3801-3817 (2010)

8. Randrianantoanina, N: Fixed point properties in Hardy spaces. J. Math. Anal. Appl. 371, 16-24 (2010)

9. Opial, Z: Weak convergence of the sequence of successive approximations for nonexpansive mappings. Bull. Am. Math. Soc. 73, 591-597 (1967)

10. Jiménez-Melado, A: Stability of weak normal structure in James quasi reflexive spaces. Bull. Aust. Math. Soc. 46(3), 367-372 (1992)

11. Domínguez-Benavides, T, García-Falset, J, Japón, MA: The $\tau$-fixed point property for nonexpansive mappings. Abstr. Appl. Anal. 3(3-4), 343-362 (1998)

12. Ayerbe, JM, Domínguez Benavides, T, López Acedo, G: Measures of Noncompactness in Metric Fixed Point Theory. Oper. Theory Adv. Appl. Birkhäuser, Basel (1997)

13. Lau, AT-M, Ülger, A: Some geometric properties on the Fourier and Fourier-Stieltjes algebras of locally compact groups, Arens regularity and related problems. Trans. Am. Math. Soc. 337, 321-359 (1993)

14. Takesaki, M: Theory of Operator Algebras I. Springer, New York (1979)

15. Lindenstrausss, J, Tzafriri, L: Classical Banach Spaces I. Springer, Berlin (1979)

16. Barrera-Cuevas, A, Japón, MA: New families of nonreflexive Banach spaces with the fixed point property. J. Math. Anal. Appl. 425(1), 349-363 (2015)

17. Lennard, $C: C_{1}$ is uniformly Kadec-Klee. Proc. Am. Math. Soc. 109, 71-77 (1990)

18. Besbes, M, Dilworth, SJ, Dowling, PN, Lennard, CJ: New convexity and fixed point properties in Hardy and Lebesgue-Bochner spaces. J. Funct. Anal. 119, 340-357 (1993)

19. Chen, S: Geometry of Orlicz Spaces. Dissertation Math. Institute of Mathematics, Warsaw (1996)

20. Musielak, J: Orlicz Spaces and Modular Spaces. Lecture Notes in Mathematics, vol. 1034. Springer, Berlin (1983)

21. Cui, Y, Hudzik, H: Maluta's coefficient and Opial's properties in Musielak-Orlicz sequence spaces equipped with the Luxemburg norm. Nonlinear Anal. 35, 475-485 (1999)

22. Thompson, HB, Cui, Y: The fixed point property in Musielak-Orlicz sequence spaces. Comment. Math. Univ. Carol. 42(2), 299-309 (2001)

23. Nakano, H: Modulared sequence spaces. Proc. Jpn. Acad. 27(9), 508-512 (1951)

24. Lin, PK: There is an equivalent norm on $\ell_{1}$ that has the fixed point property. Nonlinear Anal. 68(8), $2303-2308$ (2008)

25. Castillo-Santos, FE: Connections between geometrical and fixed point properties. Thesis, University of Newcastle (2010)

26. Kozlowski, WM: Modular Function Spaces. Dekker, New York (1998)

27. Japón, MA: Some geometric properties in modular spaces and application to fixed point theory. J. Math. Anal. Appl. 295, 576-594 (2004)

28. Domínguez-Benavides, T, Khamsi, MA, Samadi, S: Asymptotically nonexpansive mappings in modular function spaces. J. Math. Anal. Appl. 265(2), 249-263 (2002)

29. Harmand, P, Werner, D, Werner, W: M-Ideals in Banach Spaces and Banach Algebras. Lectures Notes in Mathematics, vol. 1547. Springer, Berlin (1993)

30. Pfitzner, H: L-Embedded Banach spaces and the measure topology. Isr. J. Math. 205, 421-451 (2015). doi:10.1007/s11856-014-1136-6

31. Pfitzner, $\mathrm{H}$ : Perturbations of $\ell_{1}$-copies and convergence in preduals of von Neumann algebras. J. Oper. Theory 47 145-167 (2002)

32. Japón, MA: Some fixed point results on L-embedded Banach spaces. J. Math. Anal. Appl. 272, 380-391 (2002)

33. Pfitzner, $H$ : A note on asymptotically isometric copies of $\ell_{1}$ and $c_{0}$. Proc. Am. Math. Soc. 129(5), 1367-1373 (2001) 